\title{
Expected Emotional Usefulness and Emotional Preference in Individuals with Major Depressive Disorder
}

\author{
Sunkyung Yoon ${ }^{1}$, Seung-Hwan Lee ${ }^{2,3}$, Hyang Sook Kim ${ }^{1}$ \\ ${ }^{1}$ Department of Psychology, Sogang University, Seoul, ${ }^{2}$ Department of Psychiatry, Inje University College of Medicine, Gimhae, ${ }^{3}$ Department \\ of Psychiatry, Ilsan Paik Hospital, Goyang, Korea
}

\begin{abstract}
Objective: Previous studies indicate that emotion regulation problems in major depressive disorder (MDD) may be caused by difficulties in preferring useful emotions according to their goals. We investigated expected emotional usefulness and emotional preference in individuals with MDD (MDDs) and healthy controls (HCs).

Methods: Participants were given an interpersonal scenario with two different goals (confrontation and collaboration) and rated their willingness to participate in emotion-provoking activities and the expected usefulness of a particular emotion.

Results: MDDs were similar to HCs in expected emotional usefulness but showed different patterns of emotional preference. HCs preferred happiness to negative emotions across goals whereas MDDs did not show such pattern. In addition, HCs displayed goal-appropriate preferences whereas MDDs did not prefer certain emotions for specific goals.

Conclusion: Although MDDs seemed to understand how useful an emotion can be, they did not show preference for goal-appropriate emotions. Interventions should address why MDDs have difficulty engaging in goal-appropriate emotions despite having full knowledge of the utility of emotions in achieving goals.
\end{abstract}

KEY WORDS: Depression; Emotions; Emotional aspects; Major depressive disorder.

\section{INTRODUCTION}

Major depressive disorder (MDD) is one of the most pervasive mental disorders that is characterized by augmented negative emotions and diminished positive emotions. Previous studies have consistently shown that 'emotion dysregulation' and 'psychological inflexibility' are important features and underlying mechanisms of MDD. ${ }^{1-4)}$ This raises the question of why individuals with MDD fail in emotion regulation and flexible emotional processing. Since recent studies have shown a significant relationship between emotion and perceived utility of emotion, ${ }^{5-7)}$ one hypothesis is that individuals with MDD have poor knowledge of emotions, such as an inadequate expectancy of the utility of emotions in accordance with the context.

\footnotetext{
Received: June 23, 2015 / Revised: November 24, 2015

Accepted: December 25, 2015

Address for correspondence: Hyang Sook Kim, PhD

Department of Psychology, Sogang University, 35 Baekbeom-ro, Mapo-gu, Seoul 04107, Korea

Tel: +82-2-705-8833, Fax: +82-2-705-8175

E-mail: hyangkim@sogang.ac.kr
}

According to functional approaches to emotion, there are proper contexts in which negative or positive emotions are acceptable, and healthy individuals likely modulate their emotions according to situational demand. ${ }^{8-12)}$ The desire to experience emotions based on situational goals, both consciously and unconsciously, is referred to as "emotional preference". ${ }^{13)}$ Emotional preference is the very first step in adaptive emotion regulation, ${ }^{13)}$ allowing individuals to know which emotion they want to augment or suppress. Tamir and Ford ${ }^{14)}$ found that those who prefer emotions useful for the situational demands or goals are psychologically healthier than are those who do not show flexible emotional preference depending on goals.

Considering that individuals with MDD have difficulties in regulating their emotions ${ }^{15)}$ and that emotional preference is a critical component of emotion regulation ${ }^{13)}$ individuals with MDD can be expected to have difficulties in preferring emotions useful for the situational demands. Therefore, the present study aimed to examine if individuals with MDD show deficits in emotional preference and more importantly, if they do, why they do so.

Previous studies have shown that one significant predictor of emotional preference is one's expectation of the

(a) This is an Open-Access article distributed under the terms of the Creative Commons Attribution Non-Commercial License (http://creativecommons.org/licenses/by-nc/4.0) which permits unrestricted non-commercial use, distribution, and reproduction in any medium, provided the original work is properly cited. 
utility of an emotion when dealing with a certain situational demand. ${ }^{6)}$ According to the value-expectancy mod$\mathrm{el},{ }^{16,17)}$ expectancy is a driving force for humans to move in a certain direction. In the case of emotional preference, knowing what emotion will be useful for a specific context motivates people to prefer certain emotions. Previous studies found that individuals who have a good knowledge or expectation of emotional utility display flexible and adaptive emotional preferences depending on the context. $^{6,7)}$

It has been widely known that maladaptive cognitions are a core problem of depression. ${ }^{18,19)}$ Several studies showed that depression is related to reduced emotional intelligence, which includes the ability to understand emotional knowledge and emotion. ${ }^{20)}$ In addition, Ford and Tamir $^{21)}$ found that emotional preference is significantly correlation with emotional intelligence: individuals who preferred useful emotions were more emotionally intelligent. Given the previous literature, it can be assumed that depressed individuals may lack in understating of the utility of emotion. This lack of expectation of emotional utility can also lead to inflexible emotional preference, which in turn results in maladaptive emotion regulation and inflexible emotional processing.

The present study investigated the characteristics of expected emotional usefulness and emotional preference in MDD. We hypothesized that healthy controls (HCs) would show goal-specific expected emotional usefulness and emotional preferences; specifically, they would show happiness in situations requiring collaboration and anger, in situations requiring confrontation. However, individuals with MDD (MDDs) are expected to show no goalspecific expected emotional usefulness and emotional preference, which might result in dysfunctional emotion regulation. We also predicted that for both $\mathrm{HCs}$ and MDDs there would show no goal-specific emotional preference or expected usefulness for fear, as this emotion would not be appropriate for either of the two goals (collaboration or confrontation). According to Tamir, ${ }^{13)}$ the mechanism underlying people's perceptions of contextually useful emotions can be "learned." Therefore, understanding expected emotional usefulness and emotional preference in individuals with MDD is expected to have therapeutic implications (e.g., psychoeducation of contextually useful emotions in different situations).

\section{METHODS}

\section{Participants}

MDDs were inpatients and outpatients of the Department of Psychiatry, Inje University Ilsan Paik Hospital in Goyang, Korea. HCs were recruited with flyers in the Seoul and Ilsan areas as well as through online advertisements (e.g., Facebook). A total of 54 participants (26 MDDs and $28 \mathrm{HCs}$ ) consented to participate and were administered a Korean version of the Mini-International Neuropsychiatric Interview-Plus (MINI-Plus) ${ }^{22}$ by a trained interviewer. The MINI-Plus is a short structured clinical interview that was developed according to criteria listed in the Diagnostic and Statistical Manual of Mental Disorders, 4th edition ${ }^{23)}$ and the International Statistical Classification of Diseases and Related Health Problems. ${ }^{24)}$ Patients were included only when they met criteria for MDD. Patients were excluded based on the following criteria: (1) lifetime history of psychotic symptoms, (2) lifetime diagnosis of bipolar disorders, (3) alcohol dependence/abuse in the past 6 months, and (4) observed mental retardation. Out of the $26 \mathrm{MDDs}$, three were excluded because they did not meet the criteria for current depression, and one was excluded for not finishing the behavioral tasks. As for comorbidity, five of the MDDs reported current comorbid disorders, including panic disorder, anxiety disorder not otherwise specified, and somatization disorder. All MDDs except one patient were currently taking psychotropic medication.

The required inclusion criterion for HCs was no current or history of mental disorder. HCs were also excluded when they scored higher than 14 on the Beck Depression Inventory-II (BDI-II). Three HCs were excluded for meeting this depression criterion. Thus, a total of $25 \mathrm{HCs}$ (17, female) and 22 MDDs (16, female) were included in the analysis.

\section{Measures}

Rating of current emotional state: Current emotional state was measured to control the possibility that emotional preference was affected by current emotional states. Participants were asked to rate to what extent they experienced feelings related to anger, happiness, and fear at the time of the experiment on a 7-point Likert scale, as used by Tamir et al. ${ }^{7)}$ To measure anger, a useful emotion in a confrontation situation, the averages of irritated, aggressive, angry, and hostile feelings were computed. For happiness, which can be useful for the goal of collaboration, the averages of happy, cheerful, and excited feelings were used. 
As for fear, an inappropriate emotion for both confrontation and collaboration, the average scores of fearful, worried, and distressed feelings were used. To avoid giving participants the impression that this study assessed emotional functioning, non-emotional words such as hungry, tired, and focused were added. For the statistical analyses, we used centered scores of current emotional experience, as done previously. ${ }^{7}$

Emotional preference tasks: These tasks were administered according to a previous study on emotional preference. ${ }^{7)}$ To motivate participants, a virtual task that included an online chat forum with another researcher for $1 \mathrm{mi}-$ nute was used. Participants were told they would engage in an online chat with another researcher after being given a scenario and instructions for two goals (collaboration and confrontation). Participants performed both goal tasks in a counterbalanced order. The scenario is described below:

"You are the president of a company and you will be chatting online with another researcher who will play the role of an employee. The employee is in charge of the company's investment plan. Currently, the employee has been pushing for a new investment plan, which may lead the company to miss an important opportunity for investment."

The two goals were as follows: the confrontation goal involved forcing the employee to give up on this new plan and implement the company's investment plan immediately. The collaboration goal involved discussing a way to produce an optimal investment plan with the employee. In addition, participants rated how much they wanted to be involved in particular emotion-inducing activity, that is, the past-event recall task, before the role-playing task.

In the past-event recall task, participants were asked before the role-playing scenario how much they wanted to recall events in which they felt anger, happiness, or fear (e.g., "How much do you want to recall a past event in which you were angry?"). These were rated on 9-point Likert scales from 0 (not at all) to 8 (very much).

Ratings of expected usefulness of emotion: Participants rated the extent to which an emotion would be useful to attain each goal (e.g., "To what extent do you think it is useful to feel anger to confront the employee?" or "To what extent do you think it is useful to feel anger to collaborate with the employee?") on 9-point Likert scales from 0 (not at all) to 8 (very much). The expected usefulness of anger was computed by averaging scores on feeling angry and irritated, the expected usefulness of happiness was computed by averaging scores on feeling happy and lively, and fear was computed by averaging scores on feeling afraid and worried. Additionally, non-emotional adjectives, such as hungry, tired, and focused, were added as distractor items.

BDI-II: Current symptom levels of depression were assessed with the Korean version of the BDI-II. ${ }^{25,26)}$ Following the norms by Beck et al., ${ }^{25)}$ HCs who scored higher than 14 were excluded. The internal consistency (Cronbach's $\alpha$ ) of the scale in Beck et al. ${ }^{25)}$ was 0.92, while it was 0.91 in Kim et al. ${ }^{26)}$ and 0.95 in this study.

Beck Anxiety Inventory (BAI): To statistically control the influence of anxiety on the results, anxiety was assessed using the Korean-translated version of the BAI. ${ }^{27,28)}$ The internal consistency of the BAI in Beck et al. ${ }^{27)}$ was 0.92, whereas it was 0.91 in Yook and $\mathrm{Kim}^{28)}$ and 0.94 in the current study.

\section{Procedure}

Participants were told that the study examined differences in interpersonal functioning between $\mathrm{HCs}$ and MDDs. After being provided with instructions regarding the tasks, the online chat scenario was given. Participants rated current emotional experience first and were given one of the two goals randomly. The order was counterbalanced. They were instructed to rate their emotional preference in the past-event recall task after being given a goal. Participants repeated the same process with a different goal, followed by a rating of the extent to which they expected each emotion being useful for each goal. Participants then completed the BDI-II and BAI. After the behavioral tasks, all participants were assessed by a structured clinical interview. Finally, participants were debriefed and thanked. To keep the participant's motivation on the task, verbal encouragement was provided. Data of one patient with MDD was excluded due to lacking concentration in the task. All the other participants remained motivated throughout the procedure and finished the task. This study protocol has been approved by the institutional review board of Inje University Ilsan Paik Hospital, Korea. The procedure of the study is also described in Fig. 1.

\section{Statistical Analysis}

To examine expected emotional usefulness and emotion preference between MDDs and HCs, repeated-measures ANOVAs with Emotion (anger, happiness, fear) and Goal (confrontation, collaboration) as within-subjects factors and Group (MDDs, HCs) as the between-subjects factor were computed. When the sphericity assumption was violated, Huynh-Feldt correction values were used. Post-hoc comparisons were made using Bonferroni corrected $t$-test. The significance level was set at $p<0.05$. 


\section{RESULTS}

\section{Sample Characteristics}

The mean age was 48.45 (14.22, standard deviation [SD]) years in MDDs and 42.60 (12.19) years in HC. The two groups (MDDs and HC) did not differ from each other in terms of age, $t(45)=1.52, p=$ not siginificant (NS), or

\begin{tabular}{l}
\hline Presentation of scenario \\
\hline Rate current emotional states \\
\hline Presentation of the first goal \\
\hline EP: music and recall tasks \\
\hline Presentation of the second goal \\
\hline EP: music and recall tasks \\
\hline \\
\hline Rate EEU per goals \\
\hline Listen to each music again, \\
and rate emotional response \\
\hline BDI-II and BAI \\
\hline MINI-Plus screening \\
\hline
\end{tabular}

Fig. 1. The procedure of the current experiment.

$E P$, emotional preference tasks; EEU, expected emotional usefulness; BDI-II, Beck Depression Inventory-II; BAI, Beck Anxiety Inventory; MINI-Plus, Mini-International Neuropsychiatric Interview-Plus. Goals (confrontation and collaboration) were randomly presented. gender ratio, $\chi^{2}(1)=0.13, p=\mathrm{NS}$, but did differ significantly in terms of depressive and anxiety symptoms, $t(23.47)=8.16, p<0.05$ and $t(23.88)=9.00, p<0.05$, respectively. More details are presented in Table 1. In addition, there was significant group difference in current emotional state, $F(2,90)=39.41, p<0.05$. In Bonferroni post-hoc analysis, MDDs' scores for current anger and fear were higher than $\mathrm{HC}$, and score for happiness was lower than HC. Means and standards deviations of current emotional states per group are presented in Table 2.

\section{Expected Emotion Usefulness for MDDs and HCs}

First, on the contrary to our hypothesis, a significant interaction between Emotion, Goal, and Group did not emerge, $F(1.37,61.51)=0.33, p=\mathrm{NS}$. This interaction remained non-significant after controlling for current emotional experiences, $F(1.46,61.43)=0.93, p=\mathrm{NS}$, and for BAI, $F(1.38,60.89)=0.135, p=\mathrm{NS}$.

Second, there was a significant interaction between Emotion and Group, $F(1.53,68.73)=9.89, p<0.05$. However, the significant interaction between emotion and group disappeared after controlling for current emotional experience, $F(1.63,68.72)=2.77, p=\mathrm{NS}$, and BAI scores, $F(1.56,68.61)=1.65, p=\mathrm{NS}$.

Third, there was a significant interaction between emotion and goal, $F(1.37,61.51)=45.95, p<0.05$. To examine how expected emotional usefulness differed between the goal conditions, a paired $t$-test was computed. As expected, participants expected anger to be more useful in

Table 1. Sample characteristics and mean clinical symptom scores

\begin{tabular}{|c|c|c|c|c|}
\hline & MDDs & $\mathrm{HCs}$ & $t\left(x^{2}\right)$ & $p$ value \\
\hline Gender (male/female) & $6 / 16$ & $8 / 17$ & $0.13^{\dagger}$ & 0.76 \\
\hline Age $(y r)$ & $48.45 \pm 14.22$ & $42.60 \pm 12.19$ & 1.52 & 0.14 \\
\hline Age of onset $(y r)$ & $41.55 \pm 13.19$ & & & \\
\hline Duration of current episode (wk) & $22.06 \pm 19.47$ & & & \\
\hline $\mathrm{BDI}-\mathrm{II}$ & $23.64(11.29-42.00)$ & $3.44(2.92-9.00)$ & $8.16^{*}$ & $<0.001$ \\
\hline BAl & $25.32(10.93-37.00)$ & $3.64(3.64-9.00)$ & $9.00^{*}$ & $<0.001$ \\
\hline
\end{tabular}

Values are presented as number only, mean \pm standard deviation, or median (range).

MDDs, patients with major depressive disorder; HCs, healthy controls; BDI-II, Beck Depression Inventory-II; BAI, Beck Anxiety Inventory. ${ }^{\star} p<0.05$, the value for the $\chi^{2}$-test.

Table 2. Current emotional states in MDDs and HCs

\begin{tabular}{lll}
\hline & MDDs & HCs \\
\hline Current emotional state* group & & $39.41^{*}$ \\
Current anger & $3.39(0.24-4.50)$ & $1.55-23-3.25)$ \\
Current happiness & $2.82(0.26-5.33)$ & $5.08(0.24-4.33)$ \\
Current fear & $4.17(0.30-5.67)$ & $2.13(0.28-3.67)$ \\
\hline
\end{tabular}

Values are presented as mean (range).

MDDs, patients with major depressive disorder; HCs, healthy controls.

All $p$-values are $<0.001 ;{ }^{*} p<0.05$. 
the confrontation than in the collaboration, $t(44)=4.39, p$ $<0.05$, and happiness to be more useful in the collaboration than in the confrontation, $t(44)=-7.02, p<0.05$. In addition, the expected usefulness of fear did not differ between confrontation and collaboration, $t(44)=-0.11$, $p=\mathrm{NS}$. Mean scores for expected emotional usefulness for each goal for HCs and MDDs are presented in Table 3. This interaction remains significant after controlling for current emotional experiences, $F(1.46,61.43)=47.21, p$ $<0.05$, and BAI score, $F(1.38,60.89)=11.19, p<0.05$.

Finally, the main effect of Emotion was significant, $F(1.53,68.73)=51.648, p<0.05$. The post-hoc analysis showed participants across groups expected happiness most useful (mean $\pm \mathrm{SD}, 4.96 \pm 0.18$ ), anger least useful $(2.01 \pm 0.20)$ and fear in the middle $(3.00 \pm 0.31)$. This result remained significant after controlling for current emotional experiences, $F(1.63,68.72)=51.47, p<0.05, F(1.56$, 68.61) $=15.21, p<0.05$.

To sum up, on the contrary to our hypothesis, there was no significant interaction between either Emotion, Goal and Group, or between Emotion and Group. The results showed that participants across groups expected happiness as most useful, anger as least useful and fear in the middle. In addition, happiness was expected more useful in collaboration than confrontation, and anger was expected more useful in confrontation and collaboration across groups. The expectation of the utility of fear did not differ between the two goals.

\section{Emotional Preference for MDDs and HCs}

The results showed that there was a significant main effect of Emotion, $F(1.46,65.85)=20.48, p<0.05$. This main effect was modified by Group, $F(1.46,65.85)=5.69$, $p<0.05$, and Goal, $F(2,90)=12.96, p<0.05$. Furthermore, there was a significant three-way interaction between Emotion, Goal, and Group, $F(2,90)=4.29, p<0.05$. In addition, the interactions between Emotion and Group and between Emotion, Goal, and Group remained significant after controlling for current emotional experience, $F(1.60$, $67.23)=6.55, p<0.05 ; F(2,84)=3.40, p<0.05$, respectively, and for BAI scores, $F(1.51,66.57)=6.76, p<0.05$; $F(2,88)=5.20, p<0.05$, respectively. Details regarding the significant Group effect are presented below.

First, repeated-measures ANOVAs were run separately for MDDs and HCs. For MDDs, there was no significant difference in preferences between Emotions, $F(2,42)=$ 2.63, $p=\mathrm{NS}$, and no significant interaction between Emotion and Goal, $F(2,42)=1.12, p=\mathrm{NS}$. On the other hand, the results for HCs revealed a main effect of Emotion, $F(1.21,29.06)=25.84, p<0.05$, and a significant interaction between Emotion and Goal, $F(1.51$, $36.12)=20.45, p<0.05$. Post-hoc analysis revealed that HCs significantly preferred happiness to anger $(p<0.05)$ and fear $(p<0.05)$, while there was no significant difference between their anger and fear preferences $(p=\mathrm{NS})$.

Second, to further examine the interaction between Emotion and Goal for HCs, a paired $t$-test was computed. As predicted, HCs significantly preferred anger for confrontation than for collaboration, $t(24)=3.79, p<0.05$, whereas they significantly preferred happiness for collaboration than for confrontation, $t(24)=-5.34, p<0.05$. Meanwhile, preference for fear among HCs did not differ significantly between the Goal conditions, $t(24)=1.62$, $p=\mathrm{NS}$. Mean scores of emotional preference per Goal for HCs and MDDs are presented in Table 4. And the different pattern between HCs and MDDs are presented in Fig. 2.

To sum up, as predicted, there was significant interaction between both Emotion and Group, and Emotion, Goal, and Group. HCs preferred happiness to negative emotions whereas MDDs did not show particular preference for happiness. In addition, HCs significantly preferred anger for confrontation than for collaboration, and preferred happiness for collaboration than for confrontation. MDDs' preference for anger and happiness did not

Table 3. Expected emotional usefulness in MDDs and HCs

\begin{tabular}{|c|c|c|c|c|c|}
\hline \multirow{2}{*}{ Group } & \multirow{2}{*}{ Emotion } & \multicolumn{4}{|c|}{ Expected emotional usefulness } \\
\hline & & Confrontation & Collaboration & $t$ & $p$ \\
\hline \multirow[t]{3}{*}{$\mathrm{HCs}$} & Anger & $1.58 \pm 1.72$ & $0.56 \pm 0.65$ & $3.36^{*}$ & $<0.001$ \\
\hline & Happiness & $4.12 \pm 2.14$ & $6.28 \pm 1.23$ & $-5.43^{*}$ & $<0.001$ \\
\hline & Fear & $2.22 \pm 2.34$ & $2.08 \pm 1.89$ & 0.76 & 0.45 \\
\hline \multirow[t]{3}{*}{ MDDs } & Anger & $3.55 \pm 1.98$ & $2.34 \pm 1.94$ & $2.87^{\star}$ & 0.01 \\
\hline & Happiness & $3.54 \pm 1.54$ & $5.89 \pm 1.48$ & $-4.72^{*}$ & $<0.001$ \\
\hline & Fear & $3.72 \pm 2.29$ & $3.98 \pm 2.39$ & -0.70 & 0.49 \\
\hline
\end{tabular}

Values are presented as mean \pm standard deviation.

MDDs, patients with major depressive disorder; HCs, healthy controls. ${ }^{*} p<0.05$. 
Table 4. Emotional preference in MDDs and HCs

\begin{tabular}{|c|c|c|c|c|c|}
\hline \multirow{2}{*}{ Group } & \multirow{2}{*}{ Emotion } & \multicolumn{4}{|c|}{ Emotional preference } \\
\hline & & Confrontation & Collaboration & $t$ & $p$ \\
\hline \multirow[t]{3}{*}{$\mathrm{HCs}$} & Anger & $2.68 \pm 2.61$ & $1.28 \pm 1.57$ & $3.78^{*}$ & 0.00 \\
\hline & Happiness & $4.20 \pm 2.06$ & $6.32 \pm 1.44$ & $-5.34^{*}$ & 0.00 \\
\hline & Fear & $2.64 \pm 2.61$ & $2.12 \pm 2.35$ & 1.62 & 0.12 \\
\hline \multirow[t]{3}{*}{ MDDs } & Anger & $3.00 \pm 2.67$ & $2.64 \pm 2.52$ & 0.62 & 0.54 \\
\hline & Happiness & $3.27 \pm 2.68$ & $4.00 \pm 2.45$ & -1.27 & 0.22 \\
\hline & Fear & $2.23 \pm 2.20$ & $2.55 \pm 2.67$ & -0.63 & 0.54 \\
\hline
\end{tabular}

Values are presented as mean \pm standard deviation.

MDDs, patients with major depressive disorder; HCs, healthy controls. ${ }^{*} p<0.05$.

A

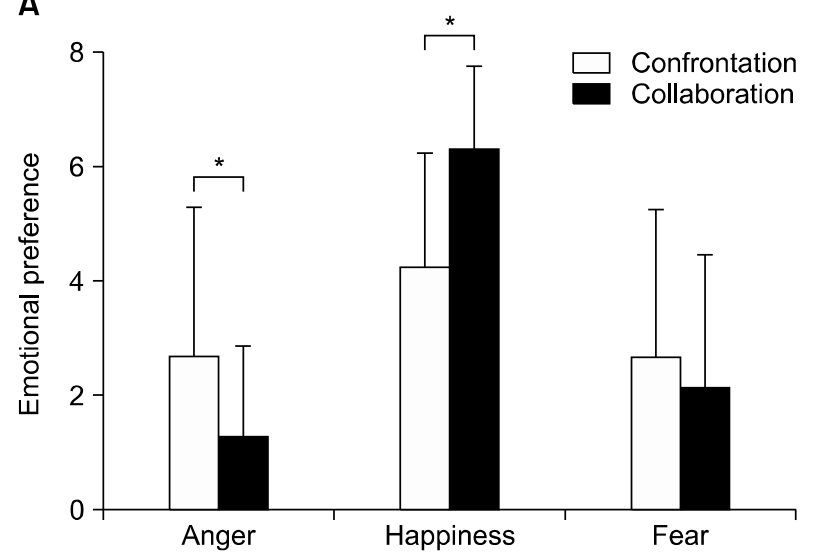

B

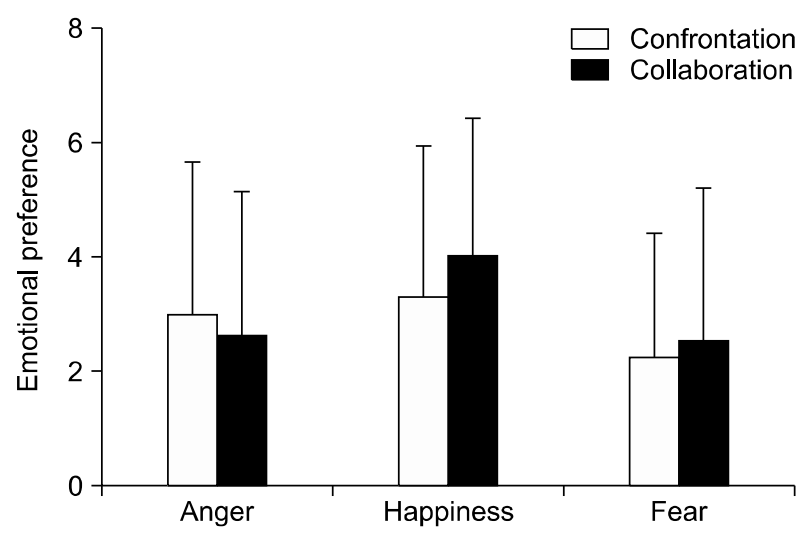

Fig. 2. Emotional preference depending on goals in $\operatorname{HCs}(A)$ and in MDDs (B). ${ }^{*} p<0.05$.

vary by goals.

\section{DISCUSSION}

The current study aimed to investigate the different patterns of expected emotional usefulness and emotional preference between MDDs and HCs. The results showed the following: 1) There was no group difference in expected emotional usefulness between MDDs and HCs. 2) Regarding emotional preference, however, MDDs did not particularly prefer happiness, whereas HCs displayed preference for happiness over negative emotions. 3) MDDs did not flexibly prefer emotions by goal, whereas HCs displayed significant emotional preferences that differed by context for both anger and happiness. 4) Finally, neither group showed goal-specific emotional preference nor expected usefulness for fear, which supports the functional approach to emotion. Fear was not a goal-related emotion for either confrontation or collaboration, and accordingly emotional preference and expected emotional usefulness for fear was not influenced by those goals. These results indicate that MDDs did not prefer useful emotions although they knew it would be useful to experience particular emotions based on context.

Contrary to our predictions, there were no group differences in expected emotional usefulness. These results indicate that MDDs were aware of the emotions that would be useful in certain situations; however, a flexible pattern of emotional preference was absent in MDDs. This is intriguing because expected emotional usefulness has been shown to be a mediator between goal and emotional preference among HCs. ${ }^{6,7)}$ Studies have also indicated that impaired cognition underlies disturbed emotion. ${ }^{5,29-31)}$ Therefore, the present study, which showed a discrepancy between expectation and actual preference, suggests that there might be a different underlying mechanism of emotional preference in patients with MDD.

Furthermore, the present study demonstrated that, regardless of group, anger was expected to be the least useful, not only compared to positive emotions but also compared to other negative emotions (e.g., fear). Expectancy of emotional utility, a conscious cognitive process, is known to be affected directly by social education. Therefore, one reason why participants perceived anger as less 
useful than fear, regardless of context, might be related to cultural characteristics. In collectivistic cultures, such as the Korean culture, individuals are socially educated to control and suppress emotions that can adversely affect harmonious relationships with others. ${ }^{32)}$ This social education regarding anger might have inculcated Koreans with the idea that anger is not useful for social interactions.

Unlike the result that MDDs have an intact ability to expect useful emotions, a different pattern of emotional preference was found between MDDs and HCs. MDDs' lack of preference for happiness is consistent with the characteristics of the disorder, which presents as diminished positive emotions as identified by the Diagnostic and Statistical Manual of Mental Disorders, fifth edition. ${ }^{33)}$ The present results are also consistent with those of previous studies on cognitive biases: non-depressed individuals tend to show unrealistic optimism (positive cognitive bias) regarding the occurrence of negative events ${ }^{34)}$ and tend to focus more on positive stimuli. ${ }^{35)}$

As for goal-specific emotional preference, the findings from the present study revealed that goal-specific emotional preference was present for HCs but not for MDDs. Tamir and Ford ${ }^{14)}$ linked contextual emotional preference to psychological flexibility, which is one of the major components of psychological well-being. ${ }^{36-38)}$ Therefore, the absence of goal-specific emotional preference found in the present study can be interpreted as a lack of flexibility in MDDs. Psychological inflexibility has been investigated in various areas such as nonverbal facial communication, ${ }^{39)}$ affective startle modulation, ${ }^{40)}$ and emotional reactivity. ${ }^{4)}$

Furthermore, deficits in goal-specific emotional preference can affect goal achievement and interpersonal functioning of MDDs. According to Tamir and Ford, ${ }^{14)}$ emotional preference is useful for actual goal achievement, which has been regarded as vital to long-term well-being, life satisfaction, and social support. ${ }^{41)}$ Therefore, the absence of contextual emotional preference in MDDs might interrupt goal achievement and adversely influence psychological well-being and interpersonal relationships.

Particularly with respect to interpersonal contexts, the present findings are compatible with Lewinsohn's theo$\mathrm{ry}^{42)}$ of depression: depressed individuals display reduced behaviors that elicit positive reinforcement from others. Emotions have a social function, which enables us to adaptively deal with situational demands relevant to other people. $^{12,43)}$ The absence of contextual emotional preference in MDDs might interfere with their social functioning. For instance, ambiguous emotional preferences might convey unclear messages to partners whom they work with. This ambiguity also evokes equivocal emotions in others who, accordingly, will not act in a way that helps to attain a particular goal. Eventually, both depressed individuals and their partners become unsatisfied with their interactions, which can worsen depression symptoms.

The current study failed to find what hampers context-appropriate emotional preference, as the knowledge of emotional usefulness was found intact in MDDs. The current findings suggested that there are factors that hamper MDDs' flexible emotional preference, other than expected emotion usefulness. In order to develop effective intervention for MDD, it would be important to find those factors and specifically target them in intervention.

One possible factor is amotivation, which is a characteristic depression symptom. Therefore, the low motivation level in depressed individuals might interfere with their ability to vary their emotional preference depending on context. In addition, MDDs' blunted reactivity to emotion and goals can also cause inflexible change of their emotional preference depending on goals. ${ }^{4)}$ Furthermore, MDDs' low self-efficacy can be another possible factor that impedes contextual emotional preference. According to the expectancy value model, ${ }^{16,17)}$ self-efficacy is a significant driving component that motivates behavior. MDDs may believe that they are incompetent in attaining goals ${ }^{44)}$ or in regulating useful emotions, ${ }^{45)}$ which may hamper their contextual emotional preference (goal-directed behavior), even though they know that the behavior would be needed to achieve the goal. Future studies can investigate these factors in relation to emotional preference. Illuminating these underlying mechanisms will further contribute to interventions for MDDs by indicating what researchers should focus on.

The present study has several limitations. First, the causal relationships between emotional preference and expected emotional usefulness were not examined due to the small sample size. Given the lack of significant group differences in expected emotional usefulness, we assumed that expected usefulness might not be the cause for the different and inflexible patterns of emotional preference in MDDs. Nevertheless, future research should test this hypothesis with a larger sample size. Second, most MDDs were using psychotropic medications. Although all MDDs were unequivocally diagnosed, the influence of medication could not be controlled. Thus, future research should assess emotional preference and expected emotional usefulness among non-medicated patients with 
MDD. Finally, although we controlled for current emotional state, it remains unclear whether the current results reflect the characteristic of MDD or influence of depressive mood. In order to better control the effect of current depressive state, future study can test this by comparing previously depressed individuals and MDDs.

In spite of these limitations, the current study contributes to elucidating emotional functioning in MDD, with this being the first study to investigate emotional preference in clinically depressed patients. The current findings revealed that MDDs lacked emotional preference for happiness across goals and flexible emotional preference depending on specific goals. However, MDDs showed the same pattern of expected emotional usefulness as HCs. These findings suggest that even though MDDs understand the usefulness of a specific emotion, they do not necessarily prefer to feel or engage in activities that would bring about that emotion. Our results also have treatment implications: educating MDDs about expectancy or knowledge of the goal-dependent function of emotion may not be effective. It would be more useful to examine other factors that yield inflexible emotional preference in MDD and to develop treatment interventions related to these factors.

\section{Acknowledgments}

A Sogang University Research Grant of 2012 (\#201210048) supported this research. This article is based on a master's thesis completed by Sunkyung Yoon at Sogang University.

\section{REFERENCES}

1. Ehring T, Tuschen-Caffier B, Schnülle J, Fischer S, Gross JJ. Emotion regulation and vulnerability to depression: spontaneous versus instructed use of emotion suppression and reappraisal. Emotion 2010;10:563-572.

2. Joormann J, Gotlib IH. Emotion regulation in depression: relation to cognitive inhibition. Cogn Emot 2010;24:281298.

3. Garnefski N, Kraaij V. Relationships between cognitive emotion regulation strategies and depressive symptoms: A comparative study of five specific samples. Personal Individ Differ 2006;40:1659-1669.

4. Bylsma LM, Morris BH, Rottenberg J. A meta-analysis of emotional reactivity in major depressive disorder. Clin Psychol Rev 2008;28:676-691.

5. Ehring T, Fischer S, Schnülle J, Bösterling A, TuschenCaffier B. Characteristics of emotion regulation in recovered depressed versus never depressed individuals. Personal Individ Differ 2008;44:1574-1584.

6. Tamir M, Ford BQ. When feeling bad is expected to be good: emotion regulation and outcome expectancies in social conflicts. Emotion 2012;12:807-816.

7. Tamir M, Ford BQ, Gilliam M. Evidence for utilitarian motives in emotion regulation. Cogn Emot 2013;27:483-
491.

8. Frijda NH. The laws of emotion. Am Psychol 1988;43: 349-358.

9. Izard CE. The psychology of emotions. New York:Springer; 1991.

10. Keltner D, Gross JJ. Functional accounts of emotions. Cogn Emot 1999;13:467-480.

11. Lazarus RS. Emotion and adaptation. New York:Oxford University Press; 1991.

12. Tooby J, Cosmides L. The evolutionary psychology of the emotions and their relationship to internal regulatory variables. In: Lewis M, Haviland-Jones JM, Barrett LF, editors. Handbook of emotions. 3rd ed. New York:Guilford;2008. p.114-137.

13. Tamir M. What do people want to feel and why? Pleasure and utility in emotion regulation. Curr Dir Psychol Sci 2009;18:101-105.

14. Tamir M, Ford BQ. Should people pursue feelings that feel good or feelings that do good? Emotional preferences and well-being. Emotion 2012;12:1061-1070.

15. Werner-Seidler A, Moulds ML. Mood repair and processing mode in depression. Emotion 2012;12:470-478.

16. Feather NT. Values, valences, expectations, and actions. $J$ Soc Issue 1992;48:109-124.

17. Nadler DA, Lawler III EE. Motivation: A diagnostic approach. In: Leavitt HJ, Pondy LR, Boje DM, editors. Readings in managerial psychology. 4th ed. Chicago, London:The University of Chicago Press;1989. p.3-19.

18. Ciesla JA, Roberts JE. Rumination, negative cognition, and their interactive effects on depressed mood. Emotion 2007; 7:555-565.

19. Coyne JC, Gotlib IH. The role of cognition in depression: a critical appraisal. Psychol Bull 1983;94:472-505.

20. Salovey P, Mayer JD. Emotional intelligence. Imagin Cogn Personal 1990;9:185-211.

21. Ford BQ, Tamir M. When getting angry is smart: emotional preferences and emotional intelligence. Emotion 2012;12: 685-689.

22. Yoo S, Kim Y, Noh J, Oh K, Kim C, Namkoong K, et al. Validity of Korean version of the MINI international neuropsychiatric interview. Anxiety Mood 2006;2:50-55.

23. American Psychiatric Association; APA Task Force on DSM-IV. Diagnostic and statistical manual of mental disorders: DSM-IV. Washington, DC:American Psychiatric Pub Inc.; 1994.

24. World Health Organization. The ICD-10 classification of mental and behavioural disorders: diagnostic criteria for research. Geneva:World Health Organization;1993.

25. Beck A, Steer R, Brown G. Manual for the beck depression inventory. San Antonio, TX:The Psychological Corporation;1996.

26. Kim M, Lee I, Lee C. The validation study I of Korean BDI-II: In female university students sample. Korean J Clin Psychol 2007;26:997-1014.

27. Beck AT, Epstein N, Brown G, Steer RA. An inventory for measuring clinical anxiety: psychometric properties. J Consult Clin Psychol 1988;56:893-897.

28. Yook S, Kim Z. A clinical study on the Korean version of Beck Anxiety Inventory: comparative study of patient and non-patient. Korean J Clin Psychol 1997;16:185-197.

29. Aldao A, Nolen-Hoeksema S. Specificity of cognitive emotion regulation strategies: a transdiagnostic examination. Behav Res Ther 2010;48:974-983.

30. Dearing KF, Gotlib IH. Interpretation of ambiguous information in girls at risk for depression. J Abnorm Child 
Psychol 2009;37:79-91.

31. Gotlib IH, Joormann J. Cognition and depression: current status and future directions. Annu Rev Clin Psychol 2010; 6:285-312.

32. Cho GH. Cultural variance in emotions. Psyhcol Sci 1997; 6:1-43. Korean.

33. American Psychiatric Association. Diagnostic and statistical manual of mental disorders: DSM-5. 5th ed. Washington, DC:American Psychiatric Association;2013.

34. Taylor SE, Brown JD. Illusion and well-being: a social psychological perspective on mental health. Psychol Bull 1988; 103:193-210.

35. Matt GE, Vázquez C, Campbell WK. Mood-congruent recall of affectively toned stimuli: A meta-analytic review. Clin Psychol Rev 1992;12:227-255.

36. Kashdan TB, Rottenberg J. Psychological flexibility as a fundamental aspect of health. Clin Psychol Rev 2010; 30:865-878.

37. Bond FW, Hayes SC, Barnes-Holmes D. Psychological flexibility, $A C T$, and organizational behavior. J Organ Behav Manage 2006;26:25-54.

38. Hayes SC, Follette VM, Linehan M. Mindfulness and acceptance: Expanding the cognitive-behavioral tradition.
New York:Guilford Press;2004.

39. Prkachin KM, Craig KD, Papageorgis D, Reith G. Nonverbal communication deficits and response to performance feedback in depression. J Abnorm Psychol 1977;86:224-234.

40. Allen NB, Trinder J, Brennan C. Affective startle modulation in clinical depression: preliminary findings. Biol Psychiatry 1999;46:542-550.

41. Brdar I, Rijavec M, Miljković D. Life goals and well-being: Are extrinsic aspirations always detrimental to well-being? Psychol Top 2009;18:317-334.

42. Lewinsohn PM, Amenson CS. Some relations between pleasant and unpleasant mood-related events and depression. J Abnorm Psychol 1978;87:644-654.

43. Keltner D, Kring AM. Emotion, social function, and psychopathology. Rev Gen Psychol 1998;2:320-342.

44. Dickson JM, Moberly NJ, Kinderman P. Depressed people are not less motivated by personal goals but are more pessimistic about attaining them. J Abnorm Psychol 2011; 120:975-980.

45. Tamir M, John OP, Srivastava S, Gross JJ. Implicit theories of emotion: affective and social outcomes across a major life transition. J Pers Soc Psychol 2007;92:731-744. 